\title{
Nutritional Support Team Approach Decreases the In-Hospital Mortality Rate after Deceased Donor Liver Transplantation
}

Sang-Oh Yun, M.D. ${ }^{1}$, Jong Man Kim, M.D., Ph.D. ${ }^{1}$, Sangjin Kim, M.D. ${ }^{1}$, Jinsoo Rhu, M.D. ${ }^{1}$, Hyun Jung Kim, M.S. ${ }^{1}$, Soo Hyun Park, M.S. ${ }^{2}$, Hyo Jung Park, M.S. ${ }^{3}$, Eunmi Gil, M.D. ${ }^{1}$, Wonseok Kang, M.D., Ph.D. ${ }^{4}$, Gyu-Seong Choi, M.D., Ph.D ${ }^{1}$, Won Hyuck Chang, M.D, Ph.D. ${ }^{5}$, Jeong-Meen Seo, M.D., Ph.D. ${ }^{1}$, Jae-Won Joh, M.D., Ph.D. ${ }^{1}$

${ }^{1}$ Department of Surgery, Samsung Medical Center, Sungkyunkwan University School of Medicine, Departments of ${ }^{2}$ Dietetics and ${ }^{3}$ Pharmaceutical Service, Samsung Medical Center, ${ }^{4}$ Division of Gastroenterology, Department of Medicine, ${ }^{5}$ Department of Physical and Rehabilitation, Samsung Medical Center, Sungkyunkwan University School of Medicine, Seoul, Korea

\begin{abstract}
Purpose: This study compared the mortality rates between a period of time without employing a nutritional support team (NST) and a period of time with an NST.

Materials and Methods: Forty-six patients underwent adult deceased donor liver transplantation (DDLT) in 2016, and their medical records were prospectively collected. All the donor recipients underwent routine enteral feeding after liver transplantation. An NST cared for twenty-one patients after September 2016. The NST consisted of transplant surgeons, hepatologists, a critical care team, a rehabilitation team, dietitians, pharmacists, and nurses. We defined the patients within the time period without an NST as the control group and those patients within the time period with an NST as the case group.

Results: There were no statistically significant differences in baseline or perioperative characteristics between the two groups. The median model for the end-stage liver disease (MELD) score was 36 (range: 21 40) for the control group and 36 (range: $23 \sim 40)$ for the case group $(P=0.596)$. The 30 -day mortality rate was $24.0 \%(6 / 25)$ for the control group, but it was $4.8 \%(1 / 20)$ for the case group. The patient survival rates at 1 -year and 2 -year were $68.0 \%$ and $64.0 \%$ in the control group and $85.7 \%$ and $81.0 \%$ in the case group, respectively. However, there were no statistically significant differences of the 30-day mortality rate and 1 2 year patient survival rate between the two groups.

Conclusion: The present study suggests that an NST should be required to prevent 30-day mortality and increase patient survival of adult DDLT patients with a high MELD score. (Surg Metab Nutr 2020;11:7-11)
\end{abstract}

Key Words: Nutritional support, Multidisciplinary approach, Liver transplantation, Malnutrition, Mortality

\section{INTRODUCTION}

Malnutrition is common among patients with chronic liver disease and patients awaiting liver transplantation (LT), because the liver is a central organ for metabolism. [1] Over $90 \%$ of patients with end-stage liver disease
(ESLD) experience protein malnutrition due to reduced protein intake, metabolic changes, long hospitalization periods, and nutritional deficiencies. In cirrhotic patients, malnourishment is associated with increased rates of complications and mortality. Inadequate protein intake in such patients also has deleterious effects such as

Received October 28, 2019. Accepted March 18, 2020

Correspondence to: Jong Man Kim, Department of Surgery, Samsung Medical Center, Sungkyunkwan University School of Medicine, 81 Irwon-ro, Gangnam-gu, Seoul 06351, Korea

Tel: +82-2-3410-1719, Fax: +82-2-3410-0040, E-mail: yjongman21@gmail.com

(c) This is an open access article distributed under the terms of the Creative Commons Attribution Non-Commercial License (http://creativecommons. org/licenses/by-nc/4.0) which permits unrestricted non-commercial use, distribution, and reproduction in any medium, provided the original work is properly cited.

Copyrights (c) The Korean Society of Surgical Metabolism and Nutrition 
hepatic encephalopathy and poor clinical outcomes.[2-5]

LT has become the treatment of choice for patients with chronic liver disease such as liver cirrhosis and hepatocellular carcinoma. Advances in surgical techniques, perioperative management and post-transplant care have greatly improved the outcomes of patients after LT; however, malnutrition significantly worsens the outcomes of LT.[6] A malnourished status prior to LT increases the rates of complications and mortality after LT [2,7,8], and many patients die after adult deceased donor liver transplantation (DDLT) because of severe malnutrition. The median model for end-stage liver disease (MELD) score was higher for DDLT patients than for living donor liver transplantation (LDLT) patients in Korea. Thus, proper nutritional status assessment and support are essential for perioperative nutritional treatment and patient survival.

Multidisciplinary approaches have proven to be highly effective for treating and managing severe disease cases. Thus, our transplantation team created a nutritional support team (NST) to manage liver transplant patients. The purpose of the present study was to compare the mortality rates between a period without an NST and a period with an NST.

\section{MATERIALS AND METHODS}

\section{Patients}

Forty-six patients underwent deceased donor liver transplantation (DDLT) at Samsung Medical Center from January to December of 2016, and their medical records were prospectively collected. We excluded LDLT patients and pediatric transplantation patients (recipients under the age of 18). The patients were divided into two groups, with the control group being defined as patients from the period without an NST $(n=25)$, and the case group being defined as patients from the period with an NST $(n=21)$. This study was approved by the Institutional Review Board (IRB) at Samsung Medical Center (SMC-201806-066), and all patients provided written informed consent prior to study enrollment.

\section{NST approach}

The NST consisted of transplant surgeons, hepatologists, a critical care team, a rehabilitation team, dietitians, pharmacists, and nurses. When a candidate patient for DDLT was admitted, a dietician would evaluate his or her nutritional status with a patient-generated subjective global assessment (SGA). Candidates for DDLT who scored over 9 points were directed to the NST, because such patients were thought to require intensive nutritional support. Patients received enteral nutrition (EN) or parenteral nutrition (PN) support during the waiting period for DDLT. Additionally, all patients consulted with the rehabilitation team to receive exercises to maintain performance and prevent muscle weakness.

\section{Enteral nutrition support}

We routinely provided EN to almost every patient via a nasogastric tube placed in the stomach for several days after DDLT. Enteral feeding was started within 12 hours after DDLT. The starting enteral nutritional amounts was $20 \mathrm{~mL} /$ hour for 12 hours. If this was well-tolerated, the infusion rate was increased to 60 to $80 \mathrm{~mL} /$ hour by postoperative day 5. A low-residual enteral liquid diet was administered. Enteral feeding was discontinued once a patient could eat more than $50 \%$ of the provided regular diet.

\section{Outcomes}

The primary endpoints were 30-day mortality rate and patient survival. The secondary endpoints were complications such as infections and acute rejection. Cytomegalovirus infection was diagnosed based on a CMV pp65 antigen-positive cell number greater than one positive cell per 200,000 white blood cells in whom the CMV antigen was not previously detectable.

\section{Statistical analysis}

All data for continuous variables are presented as median and range, while the data for categorical variables are expressed as frequency and proportion. The patient survival was estimated by the Pearson's chi-squared test 
$\left(\chi^{2}\right)$, and the groups were compared by the log-rank test. Continuous variables were compared by the Mann-Whitney $\mathrm{U}$ Test, and categorical variables were compared by Fisher's exact test. All statistical analyses were performed with SPSS 21.0 software. Differences were considered statistically significant at $\mathrm{P}<0.05$.

\section{RESULTS}

\section{Patient characteristics}

The patient characteristics are summarized in Table 1. All patients were Child-Pugh class C. The median MELD score was 36 points in both the control group and the case group. The percentage of re-transplantation was higher in the case group than in the control group (23.8\% vs. $4.0 \%$ ), as was the proportion of patients receiving continuous renal replacement therapy (CRRT) prior to DDLT ( $42.9 \%$ vs. $20.0 \%$ ); however, these differences were not statistically significant. Age, sex, body mass index, HCC co-existence, pretransplant ICU care, pretransplant ventilatory care, and hepatic encephalopathy incidence did not differ between the two groups.

The perioperative characteristics are outlined in Table 2. Recipient operation time, cold ischemic time, warm ischemic time, post-transplant intensive care unit (ICU) stay duration, and length of hospitalization did not differ between the two groups.

1) Target calorie achievement days

We estimated the target caloric intake for all patients. All patients received EN after DDLT. The control group achieved their target caloric intake in 6.4 days, while the case group achieved it in 6.7 days. There was no statistically significant difference between the two groups $(\mathrm{P}=0.377)$.

\section{2) Outcomes}

The 30-day mortality rate was $24.0 \%(6 / 25)$ in the control group but was $4.8 \%(1 / 20)$ in the case group; however, this difference was not statistically significant $(\mathrm{P}=0.070)$ (Fig. 1). The proportion of patients who contracted pneumonia within three months after DDLT was

\begin{tabular}{|c|c|c|c|}
\hline Variables & Control group $(n=25)$ & Case group $(n=21)$ & $P$ value \\
\hline Age (years) & $53(20 \sim 69)$ & $56(32 \sim 73)$ & 0.480 \\
\hline Sex & & & 0.363 \\
\hline Male & $14(56.0 \%)$ & $15(71.4 \%)$ & \\
\hline Female & $11(44.0 \%)$ & $6(28.6 \%)$ & \\
\hline Body mass index & $24.3(19.2 \sim 33.5)$ & $25.2(14.9 \sim 33.1)$ & 0.938 \\
\hline Reason for liver transplantation & & & 0.512 \\
\hline NBNC & $2(8.0 \%)$ & $1(4.8 \%)$ & \\
\hline Alcochol & $7(28.0 \%)$ & $5(23.8 \%)$ & \\
\hline HBV & $8(32.0 \%)$ & $5(23.8 \%)$ & \\
\hline $\mathrm{HCV}$ & $3(12.0 \%)$ & $1(4.8 \%)$ & \\
\hline Retransplantation & $1(4.0 \%)$ & $5(23.8 \%)$ & \\
\hline Toxic & $3(12.0 \%)$ & $2(9.5 \%)$ & \\
\hline Autoimmune & $1(4.0 \%)$ & $2(9.5 \%)$ & \\
\hline HCC co-existence & $5(20.0 \%)$ & $2(9.5 \%)$ & 0.428 \\
\hline Pretransplant ICU care & $9(36.0 \%)$ & $9(42.9 \%)$ & 0.764 \\
\hline Pretransplant ventilator & $4(16.0 \%)$ & $6(28.6 \%)$ & 0.475 \\
\hline Hepatic encephalopathy & & & 0.837 \\
\hline None & $10(40 / 0 \%)$ & $11(52.4 \%)$ & \\
\hline Grade 1 and 2 & $11(44.0 \%)$ & $3(14.3 \%)$ & \\
\hline Grade 3 and 4 & $4(16.0 \%)$ & 7 (33.3\%) & \\
\hline MELD score & $36(21 \sim 40)$ & $36(23 \sim 40)$ & 0.596 \\
\hline Hepatorenal syndrome & & & 0.198 \\
\hline None & $12(48.0 \%)$ & $8(38.1 \%)$ & \\
\hline HR without CCRT & $8(32.0 \%)$ & $4(19.0 \%)$ & \\
\hline HR with CRRT & $5(20.0 \%)$ & 9 (42.9\%) & \\
\hline
\end{tabular}

NBNC = non-B non-C; HBV = hepatitis $B$ virus; $\mathrm{HCV}=$ hepatitis $\mathrm{C}$ virus; $\mathrm{HCC}=$ hepatocellular carcinoma; ICU = intensive care unit; $\mathrm{MELD}=$ model for end-stage liver disease; $\mathrm{HR}=$ hepatorenal syndrome; CCRT = continuous renal replacement therapy. 


\begin{tabular}{|c|c|c|c|}
\hline Variables & $\begin{array}{l}\text { Control group } \\
\qquad(n=25)\end{array}$ & $\begin{array}{l}\text { Case group } \\
\qquad(n=21)\end{array}$ & $\begin{array}{c}\mathrm{P} \\
\text { value }\end{array}$ \\
\hline Recipient operative time (min) & 347 (199 490) & 335 (186 735) & 0.755 \\
\hline Cold ischemic time & 309 (203 452) & $265(40 \sim 468)$ & 0.155 \\
\hline Warm ischemic time & $29(14 \sim 68)$ & $24(13 \sim 41)$ & 0.166 \\
\hline Posttransplant ICU stay & $6(1 \sim 22)$ & $7(2 \sim 37)$ & 0.596 \\
\hline Hospitalization & $22(1 \sim 106)$ & $30(13 \sim 136)$ & 0.164 \\
\hline
\end{tabular}

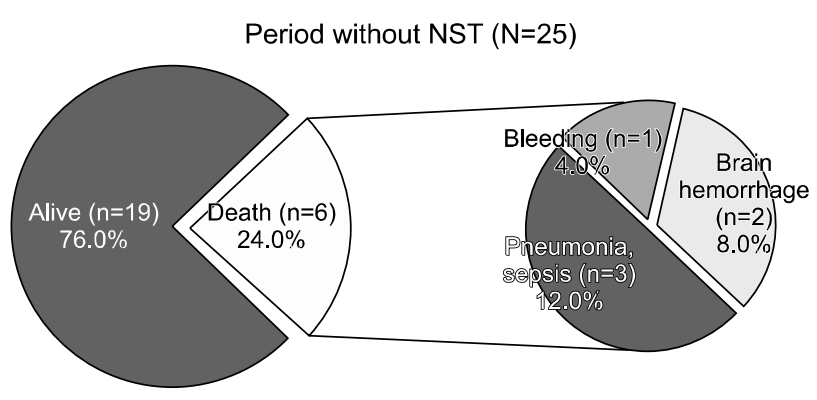

Period with NST $(\mathrm{N}=21)$

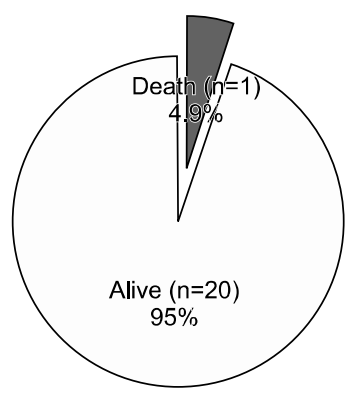

Fig. 1. In hospital mortality.

higher in the control group than in the case group (28.0\% vs. $4.8 \%$; $\mathrm{P}=0.055)$. However, the rates of infection, cytomegalovirus (CMV) infection, and acute rejection did not differ between the two groups (Table 3 ).

The 1-year and 2-year patient survival rates were $68.0 \%$ and $64.0 \%$, respectively, in the control group and were $85.7 \%$ and $81.0 \%$ in the case group. The patient survival curve was worse in the control group than in the case group, but there was no statistically significant difference between the groups $(\mathrm{P}=0.189)$.

\section{DISCUSSION}

In the present study, we observed better survival in adult DDLT patients who received NST support. There
Table 3. Complications within 3 months after adult DDLT

\begin{tabular}{lccc}
\hline & $\begin{array}{c}\text { Control group } \\
(\mathrm{n}=25)\end{array}$ & $\begin{array}{c}\text { Case group } \\
(\mathrm{n}=21)\end{array}$ & P value \\
\hline Infection & $17(68.0 \%)$ & $13(61.9 \%)$ & 0.760 \\
Pneumonia & $7(28.0 \%)$ & $1(4.8 \%)$ & 0.055 \\
Cytomegalovirus infection & $12(48.0 \%)$ & $12(57.1 \%)$ & 0.568 \\
Acute rejection & $2(8.0 \%)$ & $2(9.5 \%)$ & 0.855 \\
\hline
\end{tabular}

are several plausible explanations for this improvement. An NST can alter the direction of patient care by improving the patient's nutritional status in the perioperative period and changing their treatment recommendations.

Those who received NST support had different baseline characteristics than those who did not. The proportions of patients requiring re-transplantation due to graft failure or CCRT due to severe hepatorenal syndrome in the pre-transplant period were greater in the NST support (case) group than in the control group. The 30-day mortality and patient survival rates were better in the case group than in the control group. In addition, the incidence of pneumonia was lower in the case group than in the control group. However, the differences in 30-day mortality, patient survival, and pneumonia incidence were not statistically significant. Our study suggests that NST support is more beneficial when DDLT patients have high MELD score and many complications.

A multidisciplinary approach with coordinated surgical, medical and nutritional management is needed to improve the outcomes of LT. A previous study indicated that managing the nutritional status of patients undergoing transplantation is complex and requires specialized knowledge. Nutritional counselling and support are now recognized as necessary to reduce the severity of nutritional impairment in such patients; however, physicians have minimal training and experience in the area of nutritional support. A multidisciplinary team focused on nutritional support is the best way to solve the complex problems arising in patients undergoing transplantation. Such teams generally consist of health care professionals specializing in each area of patient care, such as dietitians, nurses, physicians, and pharmacists.[9] Merli et al. [6] reported that dietary counseling may only benefit clin- 
ically stable patients who are followed for an adequate period of time while on the waiting list. Our approach to improving liver transplantation outcomes included assessing the admitted patients awaiting transplantation; selecting the patients who needed nutritional support pre-, peri- and post-operatively; calculating the exact caloric requirement of each patient; and following up to assess compliance. This approach improved outcomes in the NST period.

However, our study had several limitations. First, because the sample size was small, its statistical power of the study was limited and statistical outcomes including survival rates and target calorie achievement days between two groups were not different although death rate in the group without NST was higher. Second, improvement in protein supply or managing EN intolerance might be needed to measure the effect of NST approach meaning NST had an effect on survival rates, but we had difficulty in analyzing quantitatively in those variables. Furthermore, in assessing patient nutritional state, we did not assess specific variables within the subjective global assessment (SGA) or measure parameters such as midarm muscle circumference (MAMC); only BMI was recorded. Only 7 patients recorded PG-SGA and the others did not because most of the patients were nutritionally healthy $(n=28)$, one patient denied to do PG-SGA, and 10 patients were unable to answer the form due to their medical condition (e.g. hepatic encephalopathy). However, the complications have been lowered, which is clearly possible because of the NST effect. Third, we did not evaluate performance status, which is an important variable for estimating the postoperative recovery of patients.

In conclusion, the present study suggests that an NST prevents 30-day mortality and increases patient survival in adult DDLT patients with high MELD score. Therefore, NST consultation should be implemented to increase the survival of such patients or patients with many complications, through accurate assessment and management of nutritional status.

\section{CONFLICTS OF INTEREST}

The authors of this manuscript have no conflicts of interest to disclose.

\section{AUTHOR'S CONTRIBUTION}

Sang-Oh Yun: literature search, data interpretation, and writing.

Jong Man Kim: design, data acquisition, analysis, interpretation.

Hyung Joo Kim, Soo Hyun Park, and Hyo Jung Park: data acquisition and interpretation.

Sangjin Kim, Jinsoo Rhu, and Eunmi Gil: data acquisition and interpretation.

Wonseok Kang, Gyu-Seong Choi, and Won Hyuck Chang: data interpretation and writing.

Jeong-Meen Seo and Jae-Won Joh: design, data interpretation, and writing.

\section{REFERENCES}

1. Kim JM, Joh JW, Kim HJ, Kim SH, Rha M, Sinn DH, et al. Early enteral feeding after living donor liver transplantation prevents infectious complications: a prospective pilot study. Medicine (Baltimore) 2015;94:e1771.

2. Alberino F, Gatta A, Amodio P, Merkel C, Di Pascoli L, Boffo $G$, et al. Nutrition and survival in patients with liver cirrhosis. Nutrition 2001;17:445-50.

3. Henkel AS, Buchman AL. Nutritional support in patients with chronic liver disease. Nat Clin Pract Gastroenterol Hepatol 2006;3:202-9.

4. Hammad A, Kaido T, Aliyev V, Mandato C, Uemoto S. Nutritional therapy in liver transplantation. Nutrients 2017;9:E1126.

5. Córdoba J, López-Hellín J, Planas M, Sabín P, Sanpedro F, Castro F, et al. Normal protein diet for episodic hepatic encephalopathy: results of a randomized study. J Hepatol 2004;41: 38-43.

6. Merli M, Giusto M, Gentili F, Novelli G, Ferretti G, Riggio O, et al. Nutritional status: its influence on the outcome of patients undergoing liver transplantation. Liver Int 2010;30:208-14.

7. Selberg O, Böttcher J, Tusch G, Pichlmayr R, Henkel E, Müller MJ. Identification of high- and low-risk patients before liver transplantation: a prospective cohort study of nutritional and metabolic parameters in 150 patients. Hepatology 1997;25:652-7.

8. Pikul J, Sharpe MD, Lowndes R, Ghent CN. Degree of preoperative malnutrition is predictive of postoperative morbidity and mortality in liver transplant recipients. Transplantation 1994:57:469-72.

9. Hagiwara S, Mori T, Tuchiya H, Sato S, Higa M, Watahiki M, et al. Multidisciplinary nutritional support for autologous hematopoietic stem cell transplantation: a cost-benefit analysis. Nutrition 2011;27:1112-7. 